\title{
BRASSINOSTEROID CONTROL OF SHOOT GRAVITROPISM INTERACTS WITH ETHYLENE AND DEPENDS ON AUXIN SIGNALING COMPONENTS ${ }^{1}$
}

\author{
Filip Vandenbussche ${ }^{2}$, Pieter Callebert ${ }^{2}$, Petra Zadnikova ${ }^{3}$, Eva Benkova $^{3}$, \\ and Dominique Van Der Straeten 2,4
}

\begin{abstract}
${ }^{2}$ Laboratory of Functional Plant Biology, Department of Physiology, Ghent University, Karel Lodewijk Ledeganckstraat 35, B-9000 Gent, Belgium; and ${ }^{3}$ VIB Department of Plant Systems Biology, Ghent University, Technologiepark 927, 9052 Gent, Belgium

- Premise of the study: To reach favorable conditions for photosynthesis, seedlings grow upward when deprived of light upon underground germination. To direct their growth, they use their negative gravitropic capacity. Negative gravitropism is under tight control of multiple hormones.

- Methods: By counting the number of standing plants in a population or by real time monitoring of the reorientation of gravistimulated seedlings of Arabidopsis thaliana, we evaluated the negative gravitropism of ethylene or brassinosteroid (BR) treated plants. Meta-analysis of transcriptomic data on AUX/IAA genes was gathered, and subsequent mutant analysis was performed.

- Key results: Ethylene and BR have opposite effects in regulating shoot gravitropism. Lack of BR enhances gravitropic reorientation in 2-d-old seedlings, whereas ethylene does not. Lack of ethylene signaling results in enhanced BR sensitivity. Ethylene and BRs regulate overlapping sets of $A U X / I A A$ genes. BRs regulate a wider range of auxin signaling components than ethylene.

- Conclusions: Upward growth in seedlings depends strongly on the internal hormonal balance. Endogenous ethylene stimulates, whereas BRs reduce negative gravitropism in a manner that depends on the function of different, yet overlapping sets of auxin signaling components.
\end{abstract}

Key words: Arabidopsis; auxin; Brassicaceae; brassinosteroid; cross talk; ethylene; gravitropism; hypocotyl; shoot.

The response of seedlings to gravity is an essential characteristic for surviving underground germination. In addition, gravitropism is crucial in redirecting shoots of crop plants after heavy storms. Upward growth of plants relies on the perception of gravity, the capacity of orientation, and the mechanical strength to support its own weight. The latter two aspects are under tight hormonal control. However, the mechanism of hormonal interactions that orchestrate negative shoot gravitropism remains largely elusive.

The first reports on gravitropism of higher plants date back to the late 19th century. Ciesielski (1872) and Darwin (1880) demonstrated the essential role of the root cap in the root graviresponse. In 1900, Haberlandt and Nemec simultaneously postulated that gravity perception is effectuated by statoliths (Greek for stationary stone), dense amyloplasts that sediment in specialized cells, the statocytes (Haberlandt, 1900; Nemec, 1900). Cytological and genetic studies proved that amyloplast sedimentation is pivotal in graviperception and necessary for a full response (Sack et al., 1986; Kiss et al., 1996; reviewed by Kiss, 2000). While the inner cells of the second tier of columella cells are major contributors in root gravitropism

\footnotetext{
${ }^{1}$ Manuscript received 3 June 2012; revision accepted 10 September 2012. The authors thank Carolina Fierro for help in screening for gene overlaps of microarray data. D.V.D.S. gratefully acknowledges the Research Foundation-Flanders (FWO-Vlaanderen, grants G.0524.07 and G.0298.09) and Ghent University for financial support. F.V. is a postdoctoral researcher of the Research Foundation-Flanders (FWO-Vlaanderen).

${ }^{4}$ Author for correspondence (e-mail: Dominique.VanDerStraeten@ ugent.be)
}

doi:10.3732/ajb.1200264
(Blancaflor et al., 1998), endodermis cells function as statocytes in the shoot. Arabidopsis shoot gravitropism 1/scarecrow (sgrl/scr) and sgr7/short root (shr) mutants, and mutants in orthologs of these genes in Pharbitis nil, that lack the endodermis in inflorescence stems, support its function in shoot graviresponse (Fukaki et al., 1996; Kitazawa et al., 2005). Perception of the directional cue by statocytes, initiates a signal transduction pathway, yet poorly understood. Changes in $\mathrm{pH}, \mathrm{Ca}^{2+}$, and $\mathrm{IP}_{3}$ concentration have been implicated in the response (Perera et al., 1999; Plieth and Trewavas, 2002). Moreover, ample evidence supports a downstream role of auxin in gravitropism. According to the Cholodny-Went theory, tropisms promote differential cellular elongation, resulting from an auxin gradient, and creating the curvature in the root or stem (Went, 1926; Cholodny, 1927). In gravistimulated seedling shoots, the highest concentration of auxin resides on the elongating side of the etiolated hypocotyl, driving differential growth (Esmon et al., 2005). Lateral auxin transport toward the lower side of the hypocotyl or stem causes differential accumulation of the hormone (Friml et al., 2002; Esmon et al., 2005; Rakusova et al., 2011). Auxin signaling depends on a group of transcriptional activators called AUXIN RESPONSE FACTORS (ARFS), which are inhibited by AUX/IAA proteins. Both $A R F \mathrm{~s}$ and $A U X / I A A$ s belong to large gene families of, respectively, 19 and 28 expressed members in Arabidopsis (Teale et al., 2006). In the presence of auxin, AUX/IAA proteins are being degraded, thus allowing ARF function. Numerous gravitropism-specific ARFs and AUX/IAA proteins have been identified (Harper et al., 2000; Tatematsu et al., 2004). ARF7, ARF19, and IAA19 all have a clear role in gravitropic growth of darkgrown hypocotyls (Tatematsu et al., 2004; Okushima et al., 

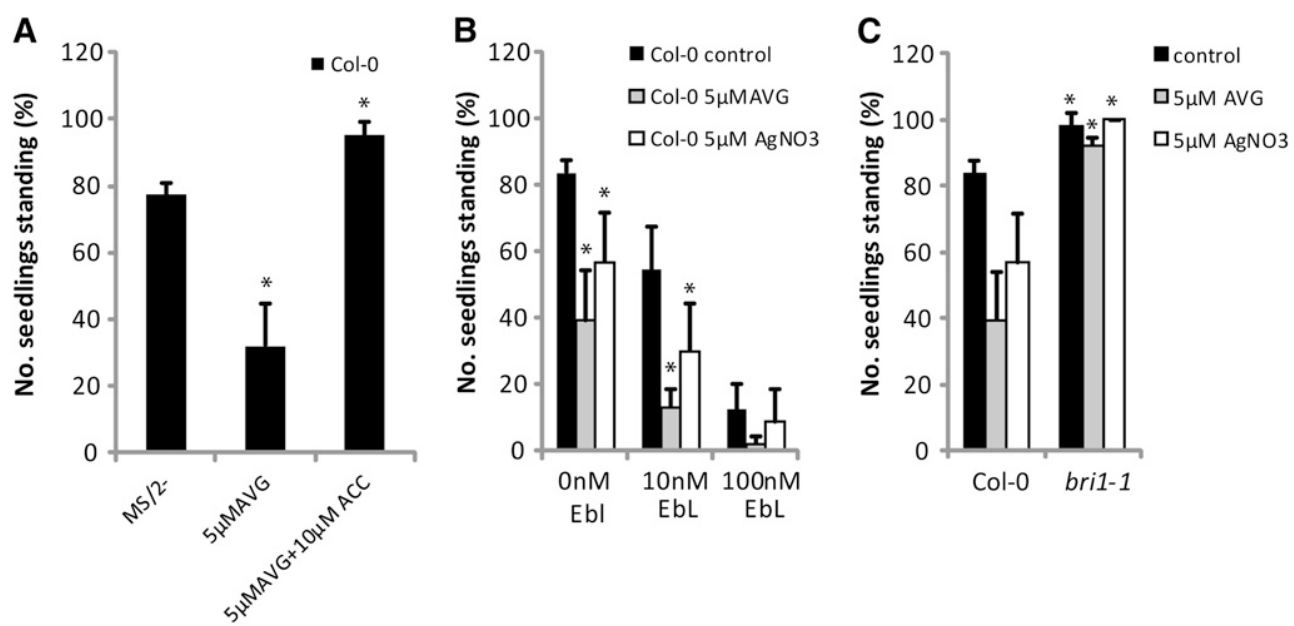

Fig. 1. Effect of ethylene on gravitropic growth in 6-d-old dark-grown seedlings of Col-0 ecotype or bri1-1 mutant of Arabidopsis thaliana. Different seedling populations were scored for their standing behavior. (A) Reduction of gravitropism by AVG treatment and reversal of the effect by ACC. Asterisks indicate statistically significant differences from the untreated control (for AVG: $\mathrm{t}=5.97, \mathrm{df}=4, P=0.019$, for AVG+ACC: $t=-5.69$, df $=4, P=0.007$ ). (B) Reduction of gravitropism by $\mathrm{AVG}, \mathrm{AgNO}_{3}$, and $\mathrm{EBL}$ in Col-0 wild type. Asterisks indicate statistically significant differences from the untreated control (for $0 \mathrm{nmol} / \mathrm{L} \mathrm{EBL}+\mathrm{AVG}: t=6.98, \mathrm{df}=8, P=0.0004$, for $0 \mathrm{nmol} / \mathrm{L} \mathrm{EBL}+\mathrm{AgNO}_{3}: t=3.92, \mathrm{df}=7, P=0.01$, for $10 \mathrm{nmol} / \mathrm{L}$ EBL$+\mathrm{AVG}: t=5.91$, $\mathrm{df}=7, P=0.005$, for $10 \mathrm{nmol} / \mathrm{L} \mathrm{EBL}+\mathrm{AgNO}_{3}: t=2.68$, $\mathrm{df}=7, P=0.03$ ). (C) Reduced effect of ethylene inhibitors $\mathrm{AVG}$ and $\mathrm{AgNO}_{3}$ in the $b r i 1-1$ mutant. Asterisks indicate statistically significant differences from the wild type Col-0 in the same conditions (for control: $t=-5.33$, df $=6, P=0.002$, for AVG: $t=-3.57, \mathrm{df}=5, P=0.03$, for $\left.\mathrm{AgNO}_{3}: t=-8.57, \mathrm{df}=5, P=0.003\right)$. Error bars represent standard deviation $(N \geq 3)$. Abbreviations for figures: ACC, 1-aminocyclopropane-1-carboxylic acid; AVG, 2-aminoethoxyvinylglycine; BR, brassinosteroid; BRZ, brassinazole; EBL, 24-epibrassinolide.

2005). However, many pleiotropic dominant mutations in other AUX/IAA genes have also been shown to interfere with shoot gravitropism (iaa3/shy2, iaa7/axr2, iaa14/slr, iaa17/axr3; reviewed by Masson et al., 2002).

Recently, brassinosteroids (BR) were identified as negative regulators of shoot gravitropism. An excess of BR suppresses, while BR deficiency promotes the upward growth of darkgrown seedlings (Nakamoto et al., 2006; Vandenbussche et al., 2011). In view of the large overlap in response gene expression between BR and auxin signaling (Nakamura et al., 2003; Nemhauser et al., 2004), it was proposed that the effect of BRs relies on auxin signaling (Vandenbussche et al., 2011).

Seedling emergence not only depends on shoot reorientation driven by the gravity vector, but also on the capability to form an apical hook to protect the meristem while the hypocotyl grows upward through the soil or mulch. The latter depends on the response to ethylene (Harpham et al., 1991). Ethylene is induced as a developmental and thigmomorphogenic signal and is necessary to keep the apical hook closed to safeguard the meristem (Harpham et al., 1991). Recent findings have placed auxins downstream of ethylene signaling in the development of the apical hook in darkness (Vandenbussche et al., 2010; Zadnikova et al., 2010). Furthermore, ethylene has been shown to promote gravitropic reorientation in light-grown seedlings (Wheeler and Salisbury, 1981; Golan et al., 1996; Guo et al., 2008). However, in Arabidopsis, ethylene has a dose-dependent dual effect on negative gravitropism in the light. Long-term exposure $(>12 \mathrm{~h})$ enhances, while short-term exposure inhibits gravibending ( $\mathrm{Li}, 2008)$.

In this study, we have focused on the tripartite interplay of BRs, ethylene, and auxins in shoot gravitropism of Arabidopsis in darkness. First, we analyzed whether ethylene plays a role in shoot gravitropism in dark-grown seedlings. Furthermore, we investigated whether BR and ethyleneregulated gravitropism are modulated by the same auxinrelated factors. We find that despite an overlap in control of gene expression, BR and ethylene also have independent ways of regulating gravitropic growth relying on different auxin-dependent factors.

\section{MATERIALS AND METHODS}

Plant material and growth media-Arabidopsis Col-0 ecotype and mutants axr2-1, ctr1-1, ein2-1, iaa6iaa19, arf7-1arf19-1, and bri1-1 were acquired from NASC (Nottingham, UK). Mutants ein3-1eill-1 and slr-1 were kind gifts from Dr. Jose Alonso and Tom Beeckman, respectively. Axr3-1 was as described (Swarup et al., 2005). Media with half strength Murashige and Skoog (Duchefa, Haarlem, Netherlands) salts were used (without addition of sucrose). Brassinazole was from TCI (Zwijndrecht, Belgium). 24-Epibrassinolide (EBL), 1aminocyclopropane-1-carboxylic acid (ACC), $\mathrm{AgNO}_{3}$, and 1- $\alpha$ (2-aminoethoxyvinyl) glycine were from Sigma (St. Louis, Missouri, USA).

Growth conditions-Seeds were surface sterilized, sown on the media, and kept for $2 \mathrm{~d}$ at $4^{\circ} \mathrm{C}$. Subsequently, they were moved to room temperature and exposed for $6 \mathrm{~h}$ to white light. For the estimation of the number of standing seedlings, plants were grown in continuous darkness for 4 to $5 \mathrm{~d}$ at $22^{\circ} \mathrm{C}$. For the reorientation assay, seedlings were grown for $2 \mathrm{~d}$ in darkness, then the square petri dishes were transferred to an IR imaging system (Vandenbussche et al., 2010) and turned over $90^{\circ}$. Photographs were taken every hour.

Determination of the number of standing seedlings and the reorientation angle-On horizontal petri dishes, seedlings were considered standing, when three quarters of their hypocotyl length, including the apical part, was not touching the medium. Averages were calculated from values for plants from at least three distinct seed batches. On vertically oriented square petri dishes that were rotated over $90^{\circ}$ for gravistimulation, horizontal seedlings were used to determine subsequent reorientation of the hypocotyls. Images were analyzed using the angle tool in the program ImageJ (National Institutes of Health, Bethesda, Maryland, USA). Angles between the horizontal and the tangential of the hypocotyl top were measured (Vandenbussche et al., 2011). All experiments were performed at least three times. Statistical analysis was based on a two-tailed Student's $t$ test.

Quantitative RT-PCR - Seeds were surface-sterilized, planted on horizontal media and kept for $2 \mathrm{~d}$ at $4^{\circ} \mathrm{C}$. Thereafter, they were moved to room temperature 



Fig. 2. Effect of increasing EBL concentrations on gravitropic growth in 6-d-old Col-0 wild type and ethylene mutants of Arabidopsis (A) in the absence of BRZ and (B) in the presence of $2 \mu \mathrm{mol} / \mathrm{L} \mathrm{BRZ}$. Asterisks indicate statistically significant differences from the wild type Col-0 grown in the same conditions (online Appendix S4). Error bars represent standard deviation $(N \geq 3)$.

for a pulse of $6 \mathrm{~h}$ of white light. Seedlings were grown for $2 \mathrm{~d}$ in darkness, then harvested. RNA was prepared using Qiagen RNeasy Plant Mini kits (Qiagen, Venlo, Netherlands). Reverse transcription was performed with a cDNA Verso kit (Thermo Scientific, Erembodegem, Belgium). Quantitative PCR was done with KAPA SYBR FAST qPCR Kit (KAPA Biosystems, Boston, Massachusetts, USA). The following primers were used: for IAA6, 5'-GGATGGTGTGCCATACATGA-3', 5'-GCATCCAGTCTCTATCCTTG-3'; for IAA 19, 5'-ACCATCTTTCAAGGCCACAC-3', 5'-GAAGCTTCGACCACGAAAGT-3'; for GAPC, 5'-AGGGGAGCAAGGAGTTAGTGC-3', 5'-GAGGGTGGTGCA-
AGAAGGTT-3'; for EIF4A, 5'-CTCATCACCACTGACCTCTTAGC-3', 5'-AACCTTCCACTTCTTCCGATAC-3'. Values were normalized to the average for $G A P C$ and EIF4A housekeeping genes.

Ethylene measurements-Ethylene was detected using a photoacoustic detector essentially as done by Vandenbussche et al. (2012). Briefly, 3-d-old seedlings grown in 10-mL vials in darkness were allowed to accumulate ethylene for $24 \mathrm{~h}$, the headspace was flushed, and ethylene was detected in the flushed air. 
TABLE 1. AUX/IAA genes and AUXIN RESPONSE FACTOR genes (ARF) transcriptionally regulated in dark-grown Arabidopsis seedlings.

\begin{tabular}{|c|c|c|c|c|}
\hline Gene ID & $\begin{array}{c}\begin{array}{c}\text { bri1.116.bzr1.1D/ } \\
\text { bri1.116 }\end{array} \\
(+\mathrm{BR} \text { signal })\end{array}$ & $\frac{\text { bri1.116/Col }}{\text { (-BR signal) }}$ & $\begin{array}{l}\text { Fold induction } \\
\frac{\text { by } \mathrm{C}_{2} \mathrm{H}_{4}}{\left(+\mathrm{C}_{2} \mathrm{H}_{4} \text { signal }\right)}\end{array}$ & Gene \\
\hline AT1G52830 & 25.98 & 0.05 & -4.5818 & IAA6 \\
\hline AT3G15540 & 24.17 & 0.04 & -2.2187 & IAA19 \\
\hline AT2G46990 & 0.56 & 1.84 & 2.1139 & IAA2O \\
\hline AT1G04240 & 7.64 & 0.19 & - & IAA3 \\
\hline AT1G15580 & 4.51 & 0.09 & - & IAA5 \\
\hline AT1G04250 & 3.65 & 0.23 & - & IAAI7 \\
\hline AT5G65670 & 3.29 & 0.57 & - & IAA9 \\
\hline AT3G23050 & 2.53 & 0.52 & - & IAA7 \\
\hline AT4G14550 & 2.16 & 0.38 & - & IAA14 \\
\hline AT1G34310 & 0.28 & 2.26 & - & $A R F 12$ \\
\hline AT2G33860 & 0.23 & 1.94 & - & ARF3 \\
\hline
\end{tabular}

\section{RESULTS}

Ethylene enhances shoot gravitropism in darkness by reducing sensitivity to brassinosteroids-Little is known about the effect of endogenous ethylene on gravitropism in darkgrown seedlings. To investigate this, wild-type seedlings were grown for $4 \mathrm{~d}$ in darkness, in the presence of the ethylene biosynthesis inhibitor 2-aminoethoxyvinylglycine (AVG). On control medium about $80 \%$ of the seedlings were standing, but only about $40 \%$ were standing on $5 \mu \mathrm{mol} / \mathrm{L} \mathrm{AVG}$ (Fig. $1 \mathrm{~A}$ and B). This effect could be reversed by simultaneous exposure to the ethylene precursor 1-aminocyclopropane-1-carboxylic acid (ACC) (Fig. 1A). Silver ions, which block ethylene perception, mimicked the AVG effect, at a somewhat less pronounced level (Fig. 1B). As a first step to investigate the cross talk between ethylene and brassinosteroids in the graviresponse in darkness, etiolated seedlings were exposed to BR combined with AVG or silver ions. 24-Epibrassinolide (EBL) caused more pronounced agravitropism (Fig. 1B; Vandenbussche et al., 2011), irrespective of the presence of ethylene inhibitors. Together these data suggest that BR and ethylene have opposite effects on gravitropism in darkness and that the BR-induced response is to a large extent independent of the ethylene signal. To further clarify the relation between ethylene and $\mathrm{BR}$, we investigated the importance of an endogenous BR signal. To this end, BR-insensitive bril-1 mutants were treated with ethylene inhibitors. Mutant bril-1 seedlings were strongly gravitropic in the presence of ethylene inhibitors, indicating that loss of gravitropism resulting from reduced ethylene signaling is dependent on the presence of an endogenous BR signal (Fig. 1C).

An EBL dose response assay on the ethylene mutant ein2-1 and double mutant ein3-1eill-1 confirmed that ethylene insensitivity leads to loss of gravitropism and that exogenous EBL treatment has a similar effect (Fig. 2A). In contrast, the constitutive ethylene response mutant $c t r l-1$ was more gravitropic than the wild type and less sensitive to exogenous EBL (significant difference at $P<0.05$ vs. $0 \mathrm{nmol} / \mathrm{L}$ EBL was only visible at $50 \mathrm{nM}$ EBL in $\operatorname{ctrl}-1$, while already at $5 \mathrm{nmol} / \mathrm{L}$ EBL for Col-0) (Fig. 2A). To determine whether ethylene affects BR biosynthesis or sensitivity, we grew seedlings on media containing $2 \mu \mathrm{mol} / \mathrm{L}$ of the BR biosynthesis inhibitor brassinazole (BRZ), which blocks endogenous BR production. In these conditions of BR deficiency, the sensitivity of ethylene mutants to BR was evaluated by adding increasing amounts of exogenous EBL (Fig. 2B). Ethylene insensitive mutants ein2-1 and ein3-1eil1-1 were more sensitive than the wild type (significant difference at $P<0.05$ vs. $0 \mathrm{nmol} / \mathrm{L}$ EBL was visible at $10 \mathrm{nmol} / \mathrm{L}$ EBL in Col-0, while already at $5 \mathrm{nmol} / \mathrm{L}$ EBL for the ethylene mutants), while the constitutive-response mutant ctrl-1 was less sensitive to BR. Hence, we can conclude that in wild-type seedlings, endogenous ethylene stimulates negative shoot gravitropism by reducing the sensitivity to BR.

Brassinosteroids and ethylene regulate the expression of common target genes-Ethylene signaling and the lack of BR response produced similar gravitropic phenotypes. It is therefore possible that ethylene and BR control the same downstream components, albeit in an opposite way. Microarray studies for ethylene and BR-related traits in dark-grown seedlings have been reported (Alonso et al., 2003; Sun et al., 2010). We compared the differentially regulated genes from these data sets and compiled the overlapping ones (Appendix S1; see Supplemental Data with the online version of this article). Since BR and ethylene act antagonistically on gravitropism, we specifically searched for genes upregulated by ethylene (Alonso et al., 2003) and downregulated by BRs (having a high level in the bril-116 mutant, Sun et al., 2010), and vice versa. Interestingly, in the presence of ethylene and in the absence of a BR signal, two AUX/IAA genes that are involved in gravitropism controlled by gibberellins (GallegoBartolomé et al., 2011), IAA6 and IAA19, are highly repressed (Table 1). The opposite pattern was found for IAA20. IAA20 is an atypical IAA protein that is not prone to IAA-mediated degradation and is therefore unlikely to be involved in auxin-controlled tropisms (Dreher et al., 2006). The BR data set also contains a large number of additional AUX/IAAs that were differentially expressed, mostly in the absence of a BR signal. Moreover, ARF3 and $A R F 12$ were also regulated by BR. Together the data show that BR controls a larger set of auxin response genes than ethylene in etiolated seedlings.

To confirm whether in our conditions IAA6 and IAAI9 are similarly affected, we analyzed RNA from 2-d-old wild-type seedlings grown in darkness in the presence of $10 \mu \mathrm{mol} / \mathrm{L}$ ACC, $100 \mathrm{nmol} / \mathrm{L}$ EBL, or $2 \mu \mathrm{mol} / \mathrm{L} \mathrm{BRZ}$ in a quantitative RT-PCR. Mutant ein2-1 seedlings were used as an ethylene-insensitive control. Low levels of IAAC and IAAI9 were found in the wild type in the presence of ACC or BRZ (Fig. 3A), consistent with the published microarray data (Table 1). We observed no differences between the wild type and the ein2-1 mutant (Fig. 3A). Analysis of the promIAA19::GUS reporter line indicated that the difference in gene expression is the result of regulation of the promoter in the hypocotyl (Fig. 3B). Upon BRZ treatment (reducing the BR signal), overall expression was reduced, while for ACC (enhancing the ethylene signal), expression in the apical hook was most affected.

Different IAAs are involved in the ethylene and the brassinosteroid response-Whereas single loss-of-function mutants in IAA genes have no phenotype because of redundancy, gain of function mutants leading to stabilized IAA proteins have defects in gravitropism (reviewed in Masson et al., 2002). To evaluate whether regulation of the IAA6 and IAA19 genes is responsible for any of the gravitropism-related phenotypes, we analyzed double loss-of-function mutants. Iaa6iaal9 seedlings were slightly less sensitive to exogenous BR (Fig. 4A), but not to AVG (Fig. 4B). This indicates that IAA6 and IAA19 have a small role in the BR response, whereas the ethylene response can be fully compensated by an IAA6-IAA19 independent mechanism. To further investigate whether this mechanism involves other IAAs, we analyzed the response of dominant negative mutations 

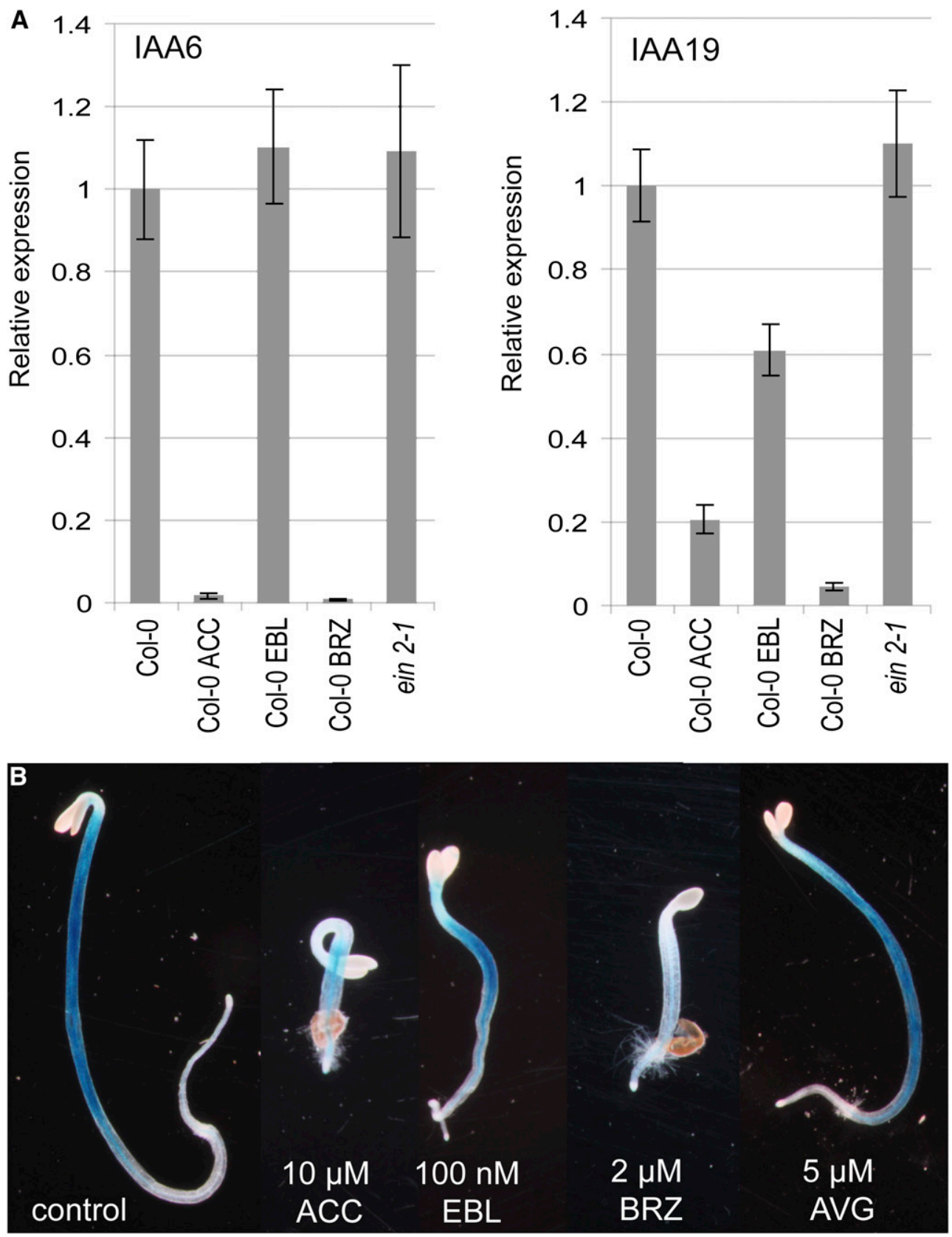

Fig. 3. Relation of the ethylene and BR response with IAA6 and IAA19. (A) qRT-PCR analysis of IAA6 and IAA19 gene expression in 2.5-d-old darkgrown Col- 0 wild type (untreated and treated with $10 \mu \mathrm{mol} / \mathrm{L}$ ACC, $100 \mathrm{nmol} / \mathrm{L}$ EBL, or $2 \mu \mathrm{mol} / \mathrm{L}$ BRZ) and untreated ein2-1 seedlings. Error bars represent standard deviation. Values were normalized to the average for GAPC and EIF4A housekeeping genes and are expressed relative to the values for untreated wild-type control. (B) Monitoring of IAA19 promoter activity by GUS staining in 2-d-old dark-grown seedlings. From left to right: untreated, treated with $10 \mu \mathrm{mol} / \mathrm{L} \mathrm{ACC,} 100 \mathrm{nmol} / \mathrm{L} \mathrm{EBL}, 2 \mu \mathrm{mol} / \mathrm{L} \mathrm{BRZ,} 5 \mu \mathrm{mol} / \mathrm{L}$ AVG.

in auxin signaling, slr-1/iaal4 and axr3-1/iaa17. The slr-1/iaal4 mutants reacted normally to BR deficiency, while showing strong resistance to ACC treatment (Fig. 4C), suggesting that IAA14 especially affects ARFs involved in the ethylene response. By contrast, axr3-1 mutants contain a stabilized version of IAA17, which is presumed to result in the constitutive inhibition of target ARFs. Analysis of axr3-1 mutant seedlings revealed strong resistance to BRZ or ACC treatment, treatments that increase shoot gravitropic response (Fig. 4D). This indicates that auxin signaling is a predominant factor in both ethylene and BR control of gravitropism.
ARF7 and ARF19 take part in the brassinosteroid and ethylene response-ARF7 interacts with IAA6 and IAA19 in a yeast two-hybrid (Y2H) assay and therefore is likely a substrate for inhibition of these IAAs (Tatematsu et al., 2004). ARF7 and its close relative ARF19 are known redundant and specific regulators of gravitropic growth in Arabidopsis thaliana; on vertical agar, dark-grown double loss-of-function mutants grow in all directions (Okushima et al., 2005).

We tested whether ARF7 and 19 are also key regulators of the response to ethylene and BRs. First, arf7arf19 seedlings were grown on increasing concentrations of ACC. Despite the fact that 

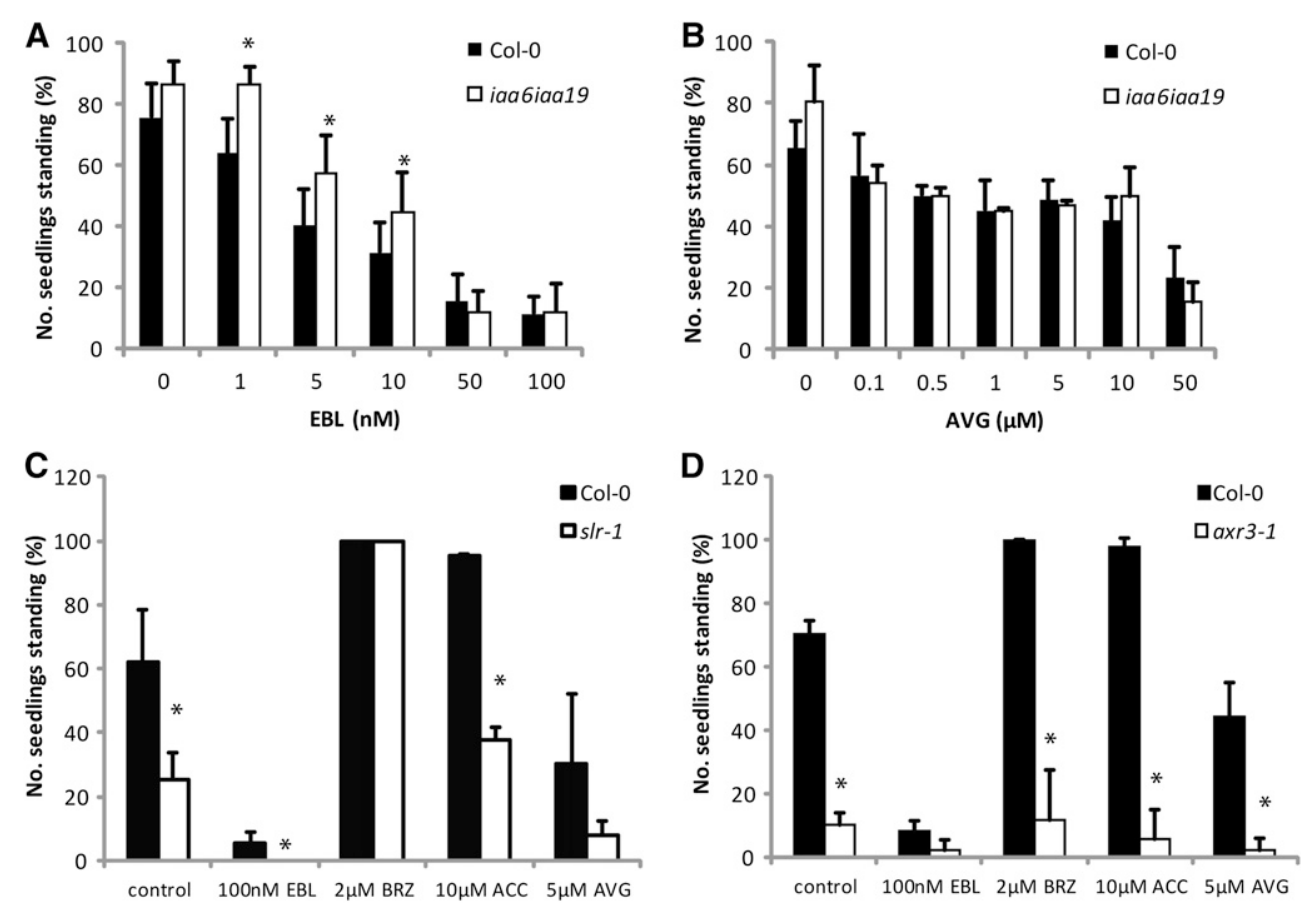

Fig. 4. Analysis of upward growth in 6-d-old dark-grown aux/iaa mutants. (A) Col-0 wild type and iaa6iaa19 double mutants of Arabidopsis treated with increasing concentrations of EBL. Asterisks indicate values statistically different from the Col- 0 wild type (for $1 \mathrm{nmol} / \mathrm{L} \mathrm{EBL:} t=-4.01, \mathrm{df}=9, P=$ 0.008 , for $5 \mathrm{nmol} / \mathrm{L}$ EBL: $t=-2.40, \mathrm{df}=9, P=0.04$, for $10 \mathrm{nmol} / \mathrm{L}$ EBL: $t=-3.05, \mathrm{df}=24, P=0.005$ ). (B) Col-0 wild type and iaa6iaa19 double mutants treated with increasing concentrations of AVG. (C) Analysis of gravitropism in the gain-of-function slr-1/iaal4 mutant in various conditions as indicated on the $x$-axis. Asterisks indicate statistically significant differences from the wild type Col-0 grown in the same conditions (for control: $t=3.48$, $\mathrm{df}=5$, $P=0.002$, for $100 \mathrm{nmol} / \mathrm{L} \mathrm{EBL}: t=7.15, \mathrm{df}=2, P=0.04$, for $10 \mu \mathrm{mol} / \mathrm{L}$ ACC: $t=20.35, \mathrm{df}=2, P=0.03$ ). (D) Analysis of gravitropism in the gain-offunction axr3-1/iaal7 mutant in various conditions as indicated on the $x$-axis. Asterisks indicate statistically significant differences from the wild type Col-0 grown in the same conditions (for control: $t=18.00, \mathrm{df}=5, P=0.00001$, for $2 \mu \mathrm{mol} / \mathrm{L} \mathrm{BRZ:} t=9.51, \mathrm{df}=3, P=0.01$, for $10 \mu \mathrm{mol} / \mathrm{L} \mathrm{ACC:} t=15.56$, $\mathrm{df}=3, P=0.001$, for $5 \mu \mathrm{mol} / \mathrm{L} \mathrm{AVG}: t=7.15, \mathrm{df}=3, P=0.002)$. Error bars represent standard deviation $(N \geq 3)$.

ACC largely rescued the arf7arf19 phenotype, over $20 \%$ of the arf7arf19 seedlings did not respond, indicating that ethylene needs ARF7 and/or ARF19 for a full response (Fig. 5A). In addition, in wild-type plants, ACC effectively counteracted the effect of 100 $\mathrm{nmol} / \mathrm{L}$ EBL, leaving more than $50 \%$ of the seedlings standing at saturating concentrations. By contrast, EBL was very efficient in reducing upward growth in the arf7arf19 double mutant even in the presence of high ACC concentrations. Hence, to counteract the BR response, ethylene is fully dependent on ARF7 and ARF19.

Second, arf7arf19 seedlings were grown on increasing concentrations of BRZ. Lack of BRs induced by high levels of BRZ could overcome the loss of gravitropism in arf7arf19 mutant seedlings by $80 \%$ (Fig. 5B), which could indicate that BRs affect other ARFs as well. However, the arf7arf19 seedlings were standing under an angle that deviated much more from the vertical than the wild type (Appendix S2; see online Supplemental Data). This is reminiscent of mutants with defects in graviperception such as pgm and scr (Vandenbussche et al., 2011). Addition of AVG to reduce the ethylene signal simultaneously with BRZ did not restore the effect of lack of BR in the wild type or the $\operatorname{arf7arf19}$ mutants. We conclude that ARF7 and ARF19 are key factors in ethylene-mediated negative gravitropic growth. However, since higher levels of ACC can rescue the double mutant phenotype, other ARFs may take over the function, or an ARF independent pathway may exist.

Downregulation of endogenous brassinosteroids improves reorientation - Shoot gravitropism as determined by upward growth is a function of the capacity of differential growth and cell wall rigidity in the hypocotyl (Vandenbussche et al., 2011). Differential growth can be uncoupled from the role of rigidity, also called gravity resistance (Hoson et al., 2005), using a reorientation assay on vertically standing petri dishes, with seedlings growing against the medium as a support. After $2 \mathrm{~d}$ of growth in darkness, the plates were turned over $90^{\circ}$, keeping the medium vertical. Untreated wild-type seedlings reorient to form an angle with the horizontal of around $45^{\circ}$ after $12 \mathrm{~h}$ (Fig. 6A). Very similar kinetics were observed in the presence of ACC, while treatment with BRZ enhanced the rate of reorientation. Untreated arf7arf19 double mutants did not react to the reorientation. Treatment with ACC caused slight reorientation, albeit far from that found in the ACCtreated wild type, indicating the importance of ARF7 and ARF19 for this response. By contrast, BRZ could rescue the reorientation in arf7arf 19 mutants to levels exceeding that of the untreated wild type, though inferior to that of the BRZ treated wild type. The latter suggests that only a small part of the BRZ response depends on functional ARF7 and/or ARF19. Hence, if auxin signaling is modulating the downstream BR control of gravitropism, other factors should be involved. To investigate this, we studied dominant negative iaa mutants that interfere with auxin signaling, by inhibiting ARFs in cells where they are expressed, using the reorientation assay. The slr-1/iaal4 mutant was unresponsive to ACC, but strongly reacted to BRZ, whereas the axr3-1/iaal7 mutant was completely insensitive to both treatments (Fig. 6B, C). Before reorientation, in all conditions used, $s l r-1$ seedlings were already bending, without a specific direction. After reorientation, slr-1 



Fig. 5. Analysis of upward growth in 6 d-old dark-grown arf7arf19 mutants of Arabdopsis. (A) Col-0 wild type and arf7arf19 double mutants treated with increasing concentrations of BRZ, in the presence or absence of $5 \mu \mathrm{mol} / \mathrm{L}$ AVG. (B) Col-0 wild type and arf7arf19 double mutants treated with increasing concentrations of ACC, in the presence or absence of $100 \mathrm{nmol} / \mathrm{L}$ EBL. Error bars represent standard deviation $(N \geq 3)$.

seedlings bent further, about half of the plants upward and the others downward, yielding a 0 average and large error bars (Fig. 6C). Together, the data demonstrate that in shoot gravitropism in darkness, both the ethylene and the BR pathways are completely dependent on auxin signaling components.

\section{DISCUSSION}

Ethylene and brassinosteroids act antagonistically in control of shoot gravitropism-We have shown that ethylene and BR have opposite effects on the upward growth of etiolated shoots. Ethylene-BR antagonism has been observed in a number of cases, for instance in roots. Ethylene reduces (Buer et al., 2006), while EBL enhances root gravitropic bending
(Kim et al., 2007). The response itself is opposite to what is found in the shoot, where ethylene enhances, while EBL reduces gravitropic growth. Interestingly, the auxin-regulated gravitropic response in root and shoot are also different. In the root graviresponse, the auxin maximum occurs at the concave (short) side, while in the shoot the maximum is located at the convex (long) side of the bending organ. The ethylene and BR effects cannot be achieved by a mere similar regulation of auxin signaling. Hormonal regulation of auxin signaling may differ in different tissues or cell types. It is conceivable that the effects of ethylene and BR go beyond the regulation of the level of auxin signaling components. Indeed, auxin distribution is also affected by ethylene and BRs, either by modulation of its synthesis or its transport (De Grauwe et al., 2005; Li et al., 2005; Buer et al., 2006; Ruzicka et al., 2007). Whereas the effect of 

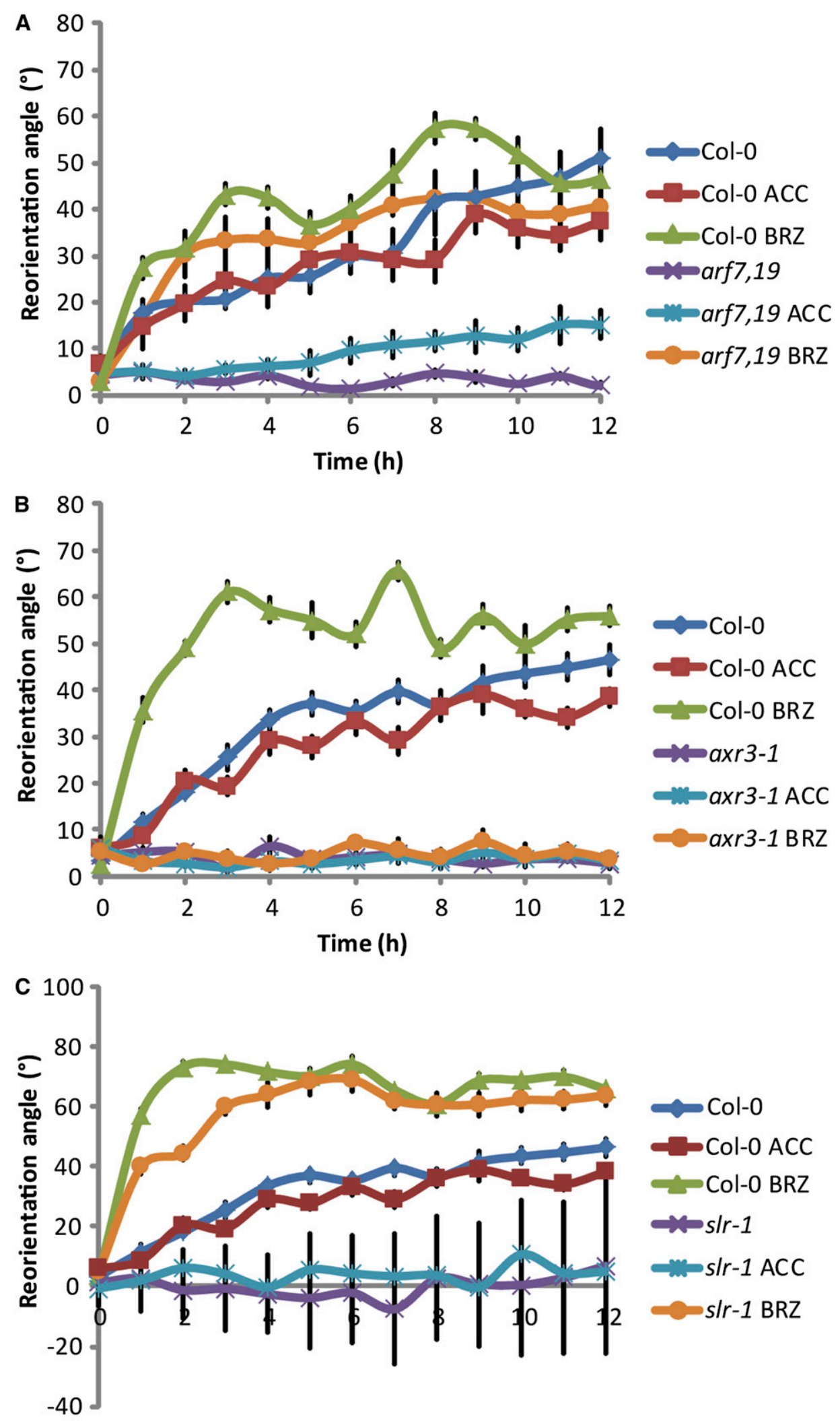

Time (h)

Fig. 6. Reorientation assay in 2-d-old dark-grown seedlings. (A) Col-0 wild type and arf7arf19 double mutants (B, C) Col-0 wild type and dominant negative aux/iaa mutants of Arabidopsis. Error bars represent standard error $(N \geq 5)$. ACC: plants grown on medium containing $10 \mu \mathrm{mol} / \mathrm{L}$ of ACC. BRZ: plants grown in the presence of $2 \mu \mathrm{mol} / \mathrm{L}$ BRZ. 
BR deficiency is consistently visible as stimulatory for upward growth in both the standing-count test and the reorientation test, the ethylene effect is visible in the standing-count test, but not in the reorientation test. These results suggest a transient regulation of graviresponse. In this case, the ethylene effect must occur earlier than the second day after germination, the point at which the reorientation assay is performed. From an ecological point of view, seedlings are likely to profit from an immediate reaction to gravity upon germination. Hence, ethylene may play an essential role in the first days after germination, supporting directional growth against the gravity vector and an apical hook that protects the cotyledons and apical meristem (Vandenbussche et al., 2010). These ethylene-dependent characteristics are of vital importance for seedling emergence from the soil (Goeschl et al., 1967; Harpham et al., 1991).

Control of AUX/IAAs and ARFs in shoot gravitropism-Control of ARFs by AUX/IAA proteins is essential for appropriate gravitropic growth. Auxins induce degradation of AUX/IAA proteins. Yet, many of the AUX/IAA genes are strongly regulated at the transcriptional level as well, most of them being auxin-inducible (Abel et al., 1995). In addition, cytokinins, gibberellins, BRs, and ethylene influence AUX/IAA transcript levels (Alonso et al., 2003; Nemhauser et al., 2004; Dello Ioio et al., 2008; Ruzicka et al., 2007; Gallego-Bartolomé et al., 2011; Fig. 3). This cross talk fine-tunes the auxin response. The other hormones may play a decisive role by tilting the balance of AUX/IAAs beyond a threshold, thus enhancing or reducing the auxin effect. Alternatively, they may regulate the basal levels of AUX/IAAs by transcriptional control in various conditions and thus transfer the threshold for auxin action on protein stability, resulting in different auxin sensitivity. For instance, BRs and auxins have strongly overlapping sets of target genes (Nemhauser et al., 2004). It appears that endogenous BRs are necessary for the expression of numerous AUX/IAAs (Fig. 3, Sun et al., 2010). Thus, BRs can increase basal expression of $A U X / I A A s$, determining the eventual auxin control on gravitropic growth via regulation of AUX/IAA-protein stability. In situations with a reduced BR signal, it becomes easier for auxins to degrade the remaining AUX/IAAs, and hence stimulate ARF activity. In the case of ethylene, the regulation of $A U X /$ $I A A s$ is not as diverse as for BR. The fact that iaa6iaal9 double mutants do not display ethylene-related phenotypes in gravitropic growth, suggests strong redundancy among the AUX/ IAAs serving as a buffer. Indeed, full dependence on AUX/IAA proteins of both the ethylene and BR response is apparent from the complete resistance of axr3/iaa17 to ACC and BRZ (Fig. 4). Alternatively, additional regulation at the level of ARFs may occur. The latter is plausible since no full dependence on the two gravitropism-specific ARFs, ARF7 and ARF19, was detected for ethylene or BR-dependent gravitropic signaling (Figs. 5, 6), which suggests that other, more pleiotropic ARFs play their role as well. It is possible that other activator ARFs, such as ARF5 or ARF8, that can interact with IAA19 in a Y2H assay (Vernoux et al., 2011) are compensating for a loss of ARF7 or ARF19 function. Hence, despite the fact that they do not appear to be regulated at the transcript level by ethylene or $\mathrm{BR}$ in the available microarray data (Alonso et al., 2003; Sun et al., 2010), it remains to be determined whether influence of these hormones occurs at the posttranscriptional level.

Our findings place auxin signaling downstream of both BR and ethylene signals in the control of shoot gravitropism in darkness (Fig. 7). However, it is possible that some effects occur
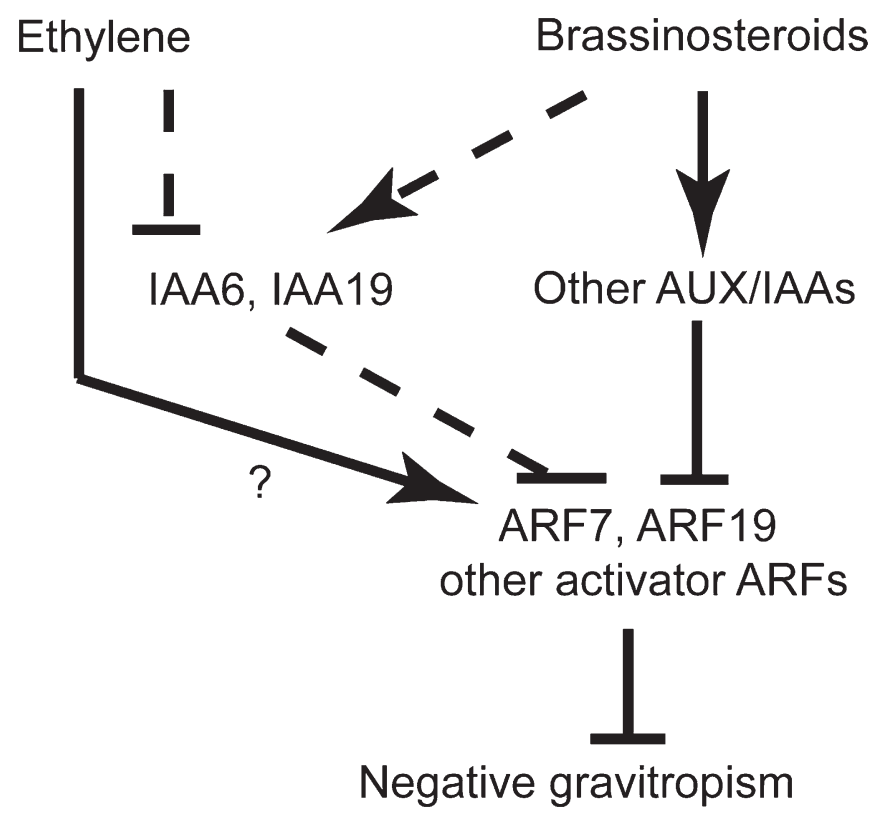

Fig. 7. Model of ethylene-BR-auxin cross talk in modulation of negative shoot gravitropism in etiolated hypocotyls. BR stimulate the expression of many IAAs, which inactivate activator auxin response factors (ARFs). As a result, negative gravitropism is inhibited. Ethylene positively affects negative gravitropism. ARF7 and ARF19 have a clear role, yet other ARF independent mechanisms may control the response. Full arrows indicate main pathways. Dashed arrows indicate minor or redundant routes. Question marks show a pathway inferred from our results, but the mechanism remains elusive. In addition, auxin-independent routes may control negative shoot gravitropism in darkness.

independent of auxin. Earlier observations have shown that cell wall rigidity also plays a part in the upward growth of Arabidopsis seedlings (Vandenbussche et al., 2011). It is conceivable that, besides its function in differential growth, auxin helps controlling overall cell wall rigidity that eventually leads to negative gravitropism. Alternatively, control of cell wall structure could indicate an additional control point that does not necessarily involve auxin signaling, but is a direct target of ethylene or BR signaling.

An ecophysiological role for hormones other than auxins in regulating gravitropism and phototropism-Shortly after germination, plants need to grow upward to reach light, often while pushing through soil or mulch. Ethylene controls thigmomorphogenesis (Takahashi and Jaffe, 1990; Yamamoto et al., 2008), and apart from being a signal that makes seedlings sturdier, also lies at the basis of an enhanced negative gravitropism (Goeschl et al., 1967; Golan et al., 1996). Indeed, wild type plants are morphogenically adapted to emerge after underground germination, whereas ethylene insensitive mutants have difficulties surviving burial (Harpham et al., 1991; Vandenbussche et al., 2010). In contrast with ethylene, BRs have an inhibitory effect on negative gravitropism in dark-grown seedlings (Vandenbussche et al., 2011). This effect seems to occur from germination onward and persists. The $\mathrm{BR}$ response may be a simple, yet undesired, consequence of the necessity of seedlings to elongate and reach the light. Indeed, BRs are elongation-stimulating hormones that are required for skotomorphogenic growth. Mutants that lack BRs have a constitutively photomorphogenic phenotype (Chory et al., 1991; Szekeres et al., 1996). In the context of seedling survival, both sufficient elongation and negative gravitropism are necessary to 
emerge after underground germination. It is therefore likely that the counteracting signals of ethylene and BR in both elongation and negative gravitropism keep a unique balance regulating upward growth. In favor of this hypothesis, ethylene production is also increased in case of an enhanced BR signal (online Appendix S3), indicating a protective control mechanism to avoid loss of sturdyness and negative gravitropism (Vandenbussche et al., 2011). Interestingly, the effect of ethylene is also visible in the light (Golan et al., 1996; Guo et al., 2008). Does BR play a role in gravitropic growth in the light? As mentioned already, light reduces BR signaling and should theoretically increase negative gravitropism. However, negative gravitropism is counteracted by the light-induced disappearance of endodermal amyloplasts, which severely reduces graviperception (Kim et al., 2011). As a result, light increases the availability of downstream, auxin-related components for use in other processes, including phototropism. Hence, downregulation of the BR signal, which confers strong reduction of many tropism inhibitory IAAs (Table 1), will assist in phototropism, rather than gravitropism in the light (Nakamoto et al., 2006). Therefore, the function of a reduction of $\mathrm{BR}$ in the light is reminiscent of the reduction of gibberellins controlling tropistic balance (Gallego-Bartolomé et al., 2011).

\section{LITERATURE CITED}

Abel, S., M. D. Nguyen, and A. Theologis. 1995. The PS-IAA4/5like family of early auxin-inducible mRNAs in Arabidopsis thaliana. Journal of Molecular Biology 251: 533-549.

Alonso, J. M., A. N. Stepanova, T. J. Leisse, C. J. Kim, H. M. Chen, P. Shinn, D. K. Stevenson, et al. 2003. Genome-wide insertional mutagenesis of Arabidopsis thaliana. Science 301: 653-657.

Blancaflor, E. B., J. M. Fasano, and S. Gilroy. 1998. Mapping the functional roles of cap cells in the response of Arabidopsis primary roots to gravity. Plant Physiology 116: 213-222.

Buer, C. S., P. Sukumar, and G. K. Muday. 2006. Ethylene modulates flavonoid accumulation and gravitropic responses in roots of Arabidopsis. Plant Physiology 140: 1384-1396.

Cholodny, N. 1927. Wuchshormone und Tropismen bei den Pflanzen. Biologisches Zentralblatt 47: 604-626.

Chory, J., P. Nagpal, and C. A. Peto. 1991. Phenotypic and geneticanalysis of $\operatorname{det} 2$, a new mutant that affects light-regulated seedling development in Arabidopsis. Plant Cell 3: 445-459.

CIESIELSKI, T. 1872. Untersuchungen über die Abwartskrummung der Wurzel. Beiträge zur Biologie der Pflanzen 1: 1-30.

Darwin, C. 1880. The power of movement in plants. John Murray, London, UK.

De Graume, L., F. Vandenbussche, O. Tietz, K. Palme, and D. Van Der Straeten. 2005. Auxin, ethylene and brassinosteroids: Tripartite control of growth in the Arabidopsis hypocotyl. Plant \& Cell Physiology 46: 827-836.

Dello Ioio, R., K. Nakamura, L. Moubayidin, S. Perilli, M. Taniguchi, M. T. Morita, T. Aoyama, Et AL. 2008. A genetic framework for the control of cell division and differentiation in the root meristem. Science 322: 1380-1384.

Dreher, K. A., J. Brown, R. E. SAw, AND J. CAllis. 2006. The Arabidopsis Aux/IAA protein family has diversified in degradation and auxin responsiveness. Plant Cell 18: 699-714.

Esmon, C. A., U. V. Pedmale, and E. Liscum. 2005. Plant tropisms: Providing the power of movement to a sessile organism. International Journal of Developmental Biology 49: 665-674.

Friml, J., J. Wiśniewska, E. BenkovÁ, K. Mendgen, and K. Palme. 2002. Lateral relocation of auxin efflux regulator PIN3 mediates tropism in Arabidopsis. Nature 415: 806-809.

Fukaki, H., H. Fujisawa, and M. Tasaka. 1996. SGR1, SGR2, and SGR3: Novel genetic loci involved in shoot gravitropism in Arabidopsis thaliana. Plant Physiology 110: 945-955.
Gallego-Bartolomé, J., C. Kami, C. Fankhauser, D. Alabadí, and M. A. BLÁzQuez. 2011. A hormonal regulatory module that provides flexibility to tropic responses. Plant Physiology 156: 1819-1825.

Goeschl, J. D., H. K. Pratt, and B. A. Bonner. 1967. An effect of light on production of ethylene and growth of plumular portion of etiolated pea seedlings. Plant Physiology 42: 1077-1080.

Golan, A., M. Tepper, E. Soudry, B. A. Horwitz, and S. Gepstein. 1996. Cytokinin, acting through ethylene, restores gravitropism to Arabidopsis seedlings grown under red light. Plant Physiology 112: 901-904.

Guo, D., X. R. Gao, H. Li, T. Zhang, G. Chen, P. B. Huang, L. J. An, ET AL. 2008. EGY1 plays a role in regulation of endodermal plastid size and number that are involved in ethylene-dependent gravitropism of light-grown Arabidopsis hypocotyls. Plant Molecular Biology 66: $345-360$.

Haberlandt, G. 1900. Ueber die Perzeption des geotropischen Reizes. Berichte der Deutschen Botanischen Gesellschaft 18: 261-272.

Harper, R. M., E. L. Stowe-Evans, D. R. Luesse, H. Muto, K. Tatematsu, M. K. Watahiki, K. Yamamoto, et al. 2000. The NPH4 locus encodes the auxin response factor ARF7, a conditional regulator of differential growth in aerial Arabidopsis tissue. Plant Cell 12: 757-770.

Harpham, N. V. J., A. W. Berry, E. M. Knee, G. Rovedahoyos, I. Raskin, I. O. SAnders, A. R. Smith, ET AL. 1991. The effect of ethylene on the growth and development of wild-type and mutant Arabidopsis thaliana (L) Heynh. Annals of Botany 68: 55-61.

Hoson, T., Y. Saito, K. Soga, and K. Wakabayashi. 2005. Signal perception, transduction, and response in gravity resistance. Another graviresponse in plants. Advances in Space Research 36: 1196-1202.

Kim, K., J. Shin, S. H. Lee, H. S. Kweon, J. N. Maloof, and G. Chol. 2011. Phytochromes inhibit hypocotyl negative gravitropism by regulating the development of endodermal amyloplasts through phytochromeinteracting factors. Proceedings of the National Academy of Sciences, USA 108: 1729-1734.

Kim, T. W., S. M. Lee, S. H. Joo, H. S. Yun, Y. Lee, P. B. Kaufman, A. KIRAKOSYAN, ET AL. 2007. Elongation and gravitropic responses of Arabidopsis roots are regulated by brassinolide and IAA. Plant, Cell \& Environment 30: 679-689.

KIss, J. Z. 2000. Mechanisms of the early phases of plant gravitropism. Critical Reviews in Plant Sciences 19: 551-573.

Kiss, J. Z., J. B. Wright, and T. Caspar. 1996. Gravitropism in roots of intermediate-starch mutants of Arabidopsis. Physiologia Plantarum 97: 237-244.

Kitazawa, D., Y. Hatakeda, M. Kamada, N. FujiI, Y. Miyazawa, A. Hoshino, S. IIDA, ET AL. 2005. Shoot circumnutation and winding movements require gravisensing cells. Proceedings of the National Academy of Sciences, USA 102: 18742-18747.

Li, L., J. Xu, Z. H. Xu, AND H. W. Xue. 2005. Brassinosteroids stimulate plant tropisms through modulation of polar auxin transport in Brassica and Arabidopsis. Plant Cell 17: 2738-2753.

LI, N. 2008. The dual-and-opposing-effect of ethylene on the negative gravitropism of Arabidopsis inflorescence stem and light-grown hypocotyls. Plant Science 175: 71-86.

Masson, P. H., M. Tasaka, M. T. Morita, C. Guan, R. Chen, and K. Boonsirichai. 2002. Arabidopsis thaliana: A model for the study of root and shoot gravitropism. Arabidopsis Book 1: e0043.

Nakamoto, D., A. Ikeura, T. Asami, and K. T. Yамамоto. 2006. Inhibition of brassinosteroid biosynthesis by either a $d$ warf4 mutation or a brassinosteroid biosynthesis inhibitor rescues defects in tropic responses of hypocotyls in the Arabidopsis mutant nonphototropic hypocotyl 4. Plant Physiology 141: 456-464.

Nakamura, A., K. Higuchi, H. Goda, M. T. Fujiwara, S. Sawa, T. Koshiba, Y. Shimada, AND S. Yoshida. 2003. Brassinolide induces IAA5, $I A A 19$, and DR5, a synthetic auxin response element in Arabidopsis, implying a cross talk point of brassinosteroid and auxin signaling. Plant Physiology 133: 1843-1853.

Nemec, B. 1900. Ueber die Art der Wahrnehmung des Schwerkraftreizes bei den Pflanzen. Berichte der Deutschen Botanischen Gesellschaft 18: 241-245. 
Nemhauser, J. L., T. C. Mockler, ANd J. Chory. 2004. Interdependency of brassinosteroid and auxin signaling in Arabidopsis. PLoS Biology 2: e258.

Okushima, Y., P. J. Overvoorde, K. Arima, J. M. Alonso, A. Chan, C. Chang, J. R. Ecker, et AL. 2005. Functional genomic analysis of the AUXIN RESPONSE FACTOR gene family members in Arabidopsis thaliana: Unique and overlapping functions of ARF7 and ARF19. Plant Cell 17: 444-463.

Perera, I. Y., I. Heilmann, and W. F. Boss. 1999. Transient and sustained increases in inositol 1,4,5-hisphosphate precede the differential growth response in gravistimulated maize pulvini. Proceedings of the National Academy of Sciences, USA 96: 5838-5843.

Plieth, C., and A. J. Trewavas. 2002. Reorientation of seedlings in the earth's gravitational field induces cytosolic calcium transients. Plant Physiology 129: 786-796.

Rakusova, H., J. Gallego-Bartolomé, M. Vanstraelen, H. S. Robert, D. Alabadí, M. A. Blázquez, E. Benkova, et al. 2011. Polarization of PIN3-dependent auxin transport for hypocotyl gravitropic response in Arabidopsis thaliana. Plant Journal 67: 817-826.

Ruzicka, K., K. Luung, S. Vanneste, R. Podhorska, T. Beeckman, J. Friml, AND E. BenKova. 2007. Ethylene regulates root growth through effects on auxin biosynthesis and transport-dependent auxin distribution. Plant Cell 19: 2197-2212.

SACK, F. D., M. M. SuYemoto, and A. C. Leopold. 1986. Amyloplast sedimentation and organelle saltation in living corn columella cells. American Journal of Botany 73: 1692-1698.

Sun, Y., X. Y. Fan, D. M. CaO, W. Q. Tang, K. He, J. Y. Zhu, J. X. He, ET AL. 2010. Integration of Brassinosteroid signal transduction with the transcription network for plant growth regulation in Arabidopsis. Developmental Cell 19: 765-777.

Swarup, R., E. M. Kramer, P. Perry, K. Knox, H. M. O. Leyser, J. Haseloff, G. T. S. Beemster, et Al. 2005. Root gravitropism requires lateral root cap and epidermal cells for transport and response to a mobile auxin signal. Nature Cell Biology 7: 1057-1065.

Szekeres, M., K. Nemeth, Z. Konczkalman, J. Mathur, A. Kauschmann, T. Altmann, G. P. Redei, Et Al. 1996. Brassinosteroids rescue the deficiency of CYP90, a cytochrome P450, controlling cell elongation and de-etiolation in Arabidopsis. Cell 85: 171-182.
TAKAHASHI, H., AND M. J. JAFFe. 1990. Thigmotropism and the modulation of tropistic curvature by mechanical perturbation in cucumber hypocotyls. Physiologia Plantarum 80: 561-567.

Tatematsu, K., S. Kumagai, H. Muto, A. Sato, M. K. Watahiki, R. M. HARPER, E. Liscum, et AL. 2004. MASSUGU2 encodes Aux/IAA19, an auxin-regulated protein that functions together with the transcriptional activator NPH4/ARF7 to regulate differential growth responses of hypocotyl and formation of lateral roots in Arabidopsis thaliana. Plant Cell 16: 379-393.

Teale, W. D., I. A. Paponov, and K. Palme. 2006. Auxin in action: Signalling, transport and the control of plant growth and development. Nature Reviews, Molecular Cell Biology 7: 847-859.

Vandenbussche, F., J. Petrasek, P. Zadnikova, K. Hoyerova, B. Pesek, V. RAZ, R. SwaruP, ET AL. 2010. The auxin influx carriers AUX1 and LAX3 are involved in auxin-ethylene interactions during apical hook development in Arabidopsis thaliana seedlings. Development 137: 597-606.

Vandenbussche, F., D. Suslov, L. De Grauwe, O. Leroux, K. Vissenberg, and D. Van Der Straeten. 2011. The role of brassinosteroids in shoot gravitropism. Plant Physiology 156: 1331-1336.

Vandenbussche, F., I. Vaseva, K. Vissenberg, and D. Van Der Straeten. 2012. Ethylene in vegetative development: A tale with a riddle. New Phytologist 194: 895-909.

Vernoux, T., G. Brunoud, E. Farcot, V. Morin, H. Van den Daele, J. Legrand, M. Oliva, ET AL. 2011. The auxin signalling network translates dynamic input into robust patterning at the shoot apex. Molecular Systems Biology 7: 508.

WENT, F. 1926. On growth accelerating substances in the coleoptile of Avena sativa. Proceedings van de Koninklijke Nederlandse Akademie der Wetenschappen 30: 10-19.

Wheeler, R. M., and F. B. Salisbury. 1981. Gravitropism in higher-plant shoots. 1. A role for ethylene. Plant Physiology 67: 686-690.

Yamamoto, C., Y. Sakata, T. TaJI, T. Baba, and S. TanaKa. 2008. Unique ethylene-regulated touch responses of Arabidopsis thaliana roots to physical hardness. Journal of Plant Research 121: 509-519.

Zadnikova, P., J. Petrasek, P. Marhavy, V. Raz, F. Vandenbussche, Z. J. Ding, K. Schwarzerova, et al. 2010. Role of PIN-mediated auxin efflux in apical hook development of Arabidopsis thaliana. Development 137: 607-617. 